Imagine a population-based cancer registry fed continually with individually linked data from warehouses contributed to by all, or nearly all, notifiers (such as hospitals and pathology laboratories). The data include information on in-patient and ambulatory surgical, chemical, biological and radiation treatment. The few notifiers who do not use a data warehouse notify through a secure Web site or completion of a notification form through their Web browser. Sophisticated case resolution software identifies new incident cases in incoming data and there is little need for human intervention. All data are coded at source and the registry's focus is quality assurance.

A new data file is released quarterly and is complete to the end of a period six months before its release. The file is immediately accessible through an easily used, analytical 'front end' on the World Wide Web.
Privacy is protected by software ensuring that disaggregation to the point of potential identifiability does not occur. Release of leading indicators of change in incidence, mortality and survival follows shortly, as does information comparing the care delivered by individual health services with best-practice care. An email alert is sent to key public health and cancer care decision-makers with a link to the new information.

Staff members spend most of their time in developing new cancer registry information products and in valueadded analysis and research using cancer registry data.

\section{This is all possible now.}

This issue of the Bulletin, the second in a five-part series on cancer, contains articles and reports describing how new and better cancer information systems are being applied in New South Wales.

\title{
STATUS REPORT ON REDEVELOPING THE NSW CENTRAL CANCER REGISTRY
}

\section{Elizabeth Tracey}

\section{Manager, Cancer Registers}

NSW Cancer Council

Since 1972, public health legislation in NSW requires that all new cases of cancer are notified. Since 1986, the NSW Central Cancer Registry (CCR) has been based at the NSW Cancer Council and has reported on the number, rates, types and distribution of cancer cases and deaths in NSW. This information has been vital for planning cancer services, and monitoring the health of the NSW population.

Currently, the NSW Public Health Act 1991 requires that hospitals and pathology laboratories notify the NSW Central Cancer Registry of people who are treated for cancer, or who have had tests that have diagnosed cancer. These notifications contain information about the patients, the type of cancer, date of diagnosis and treatment given, and are currently received by the CCR either in electronic or paper form. A number of notifications can therefore be received for the same case of cancer from different sources, and must be matched and reconciled through the process of registration. Notification from a variety of sources ensures better capture of data on as many cancer cases as possible; and, through the process of checking and registration, better quality data on each case of cancer registered.

This article describes the progress of initiatives to improve the operational efficiency of the registration process and the quality of the data received, processed, and made available for use by, the NSW Central Cancer Registry. These initiatives include:

- automated methods to validate and check data entry in hospital patient administration systems;

- improving mechanisms to receive and process data through electronic notification, and eventually through the Health Information Exchange (HIE);

- increasing the proportion of notifications received in electronic form;

- improving the processing and storage of paper notifications through the introduction of a workflow management system;

- eliminating the backlog of unprocessed notifications;

- developing a new main database to manage the data;

- making the data available in an easily accessible format.

\section{OVERVIEW OFTHE CCR REDEVELOPMENT}

The redevelopment project covers all aspects of notifying, receiving, processing, editing, extracting, analysing and reporting population-based cancer data in NSW and the ACT. The project is funded by the NSW Government and is administered by the NSW Department of Health. It aims to achieve:

- solutions that are consistent with the Department of Health Information, Management and Technology 
Strategic Plan and the NSW Central Cancer Registry Business Case ${ }^{1}$

- the removal of the backlog of notifications and assurance that reporting requirements take place within one year of notification;

- increased timeliness and quality of data;

- improved reporting of data.

\section{INITIATIVESTO IMPROVE DATA QUALITY}

Redevelopment initiatives in the following strategic areas should result in a major improvement in the quality of data and information produced by the CCR:

\section{Patient Administration System}

Currently, 40 per cent of all notifications are received electronically from Patient Administration Systems (PAS) in hospitals. These systems collect identifying and treatment data for all patients who are admitted to hospitals throughout the State. In the past, PAS systems have included extra fields in a cancer module, to collect data that is relevant only to patients with cancer.

With the development of new PAS systems in NSW hospitals, the CCR redevelopment process is seeking to: ensure that the cancer module is included and improved upon so that all relevant cases of cancer are notified to the CCR; and to automate data checks at the source of data entry. The business requirements of the Registry have been given to the suppliers of PAS systems to ensure that the necessary edits and validation are incorporated in the design of present and future PAS systems. ${ }^{2}$ These specifications will require the compulsory completion of the cancer module by the expert medical coder, using relevant disease codes. Currently the completion of the module is at the discretion of these coders.

\section{Data dictionary}

Other initiatives include defining the cancer registry data items in a new cancer-specific data dictionary, which is available on the Department of Health's Web site. ${ }^{3}$ The 11 cancer-specific data items, which are included in the cancer module of the PAS systems, are defined in this data dictionary, along with other data items notified to the CCR that are also used in other data collections such as the Inpatient Statistics Collection (ISC).

The development of the data dictionary has involved reviewing all data collected, and has provided the opportunity to address existing anomalies in data definitions used by the CCR and the Health Department in other collections, such as the ISC. For example, the codes that the CCR uses to identify institutions, and the country of birth of patients, have been changed so that they are the same as those used by the Department of
Health. New codes have also been allocated for private pathology laboratories. In future, the CCR will only use codes allocated by the Department of Health. The postcode and locality tables have also been updated and an automatic coding facility (National Locality Index Grouper) has been introduced to allocate residential addresses to local government areas and area health services.

The alignment to the ISC, and the introduction of processes to ensure that data codes continue to be aligned with those of the Department of Health, will help to ensure the consistency and quality of the CCR data.

\section{Health Information Exchange}

By June 2001, cancer notifications will be received from the Health Information Exchange (HIE). ${ }^{4}$ The HIE comprises individual data warehouses of each area health services and the Department of Health. The warehouses are repositories of historic information collected from disparate operational systems throughout the health system, including the PAS systems. The inclusion of the cancer module data will further improve the quality of the data received by the CCR in a number of ways. First, notifications that have incomplete codes on cancer type will be flagged and automatically sent back to the facilities to update. Second, output from the HIE can be presented in a consistent format that can be imported directly into the CCR database.

In time, notifications from pathology laboratories may also be incorporated into the HIE. In the short term, it is hoped that these notifications will be received in an electronic format that will allow for data from different pathology information systems to be received and used by the CCR. An Australian standard has been developed for the electronic transmission of pathology records, but further investigation is required to determine if this can be applied in practice using existing pathology systems. Preliminary discussions have been held with pathology laboratories and a pilot study is being planned.

\section{Workflow management system}

To process the remaining written notifications, and to streamline the processing of notifications by the CCR, a workflow management system is being introduced. This system will include imaging facilities that capture paper notifications in different formats as electronic images (data in the images can be read but not manipulated). This will increase the efficiency and accuracy of processing incoming paper records. The imaged records can then be read on computer screens for processing purposes, will be much more accessible than paper records, and will eliminate the need for additional, long-term storage of paper. 


\section{MAIN DATABASE DEVELOPMENT}

The present database has been fully documented, including data models that describe the relationship between types of data, and the data dictionary that defines the individual data items. User requirements for the new database have been specified, and functional and technical specifications are being prepared. An audit of common reasons why records fail to load onto the current database will inform the development of the matching and code resolution algorithms for the new database. These algorithms ensure not only that people are correctly linked, but also that new notifications are correctly identified as new cancers or as re-notifications of cancers already known to the database.

\section{PROCESSING THE BACKLOG}

As at July 2000, there was a backlog of approximately 120,000 paper notifications filed alphabetically that require processing. An audit of CCR processes was undertaken to estimate average times for processing mail, sorting, coding, data entry and editing to estimate the resources required in order to eliminate the backlog in 12 months. ${ }^{6}$ The audit results will also be used to evaluate the effect of the introduction of a workflow management system. Additional staff have been appointed and the following timetable has been set: the 1998 Cancer Registry report produced by August 2001, the 1999 report by December 2001 and the 2000 report by June 2002. Monitoring of the progress indicates that the Registry will deliver these reports on time or earlier.

\section{REPORTING}

Consultations were held to gain advice from members of the Epidemiology Special Interest Group, Directors of Health Service Development, the Central Cancer Registry, and staff of the Cancer Epidemiology Research Unit, on a set of standards for reports. An improved Statistical Analysis Software (SAS) reporting module is nearing completion, which has a userfriendly series of drop down menus that present CCR data geographically, demographically, clinically, and by time period.

Up-to-date releases of an appropriately restricted CCR database, from which data that may identify an individual has been removed, will be produced quarterly for use with the annual reporting module. The quarterly module will be made available to authorised staff of the Cancer Council's Cancer Research and Registers Division. It may also be possible to make it more widely available to public health personnel in the NSW health system, through the Health Outcomes Information and Statistical Toolkit (HOIST), which is a public health data warehouse developed and maintained by the Epidemiology and Surveillance Branch of the NSW Department of Health.

A Web-based version of the reporting module, providing access to a large number of pre-prepared tables, is also being developed for the use of the general public. Both tables and graphs of data in age-specific categories and in age-standardised and crude form will be available, as well as new measures, such as person years of life lost due to cancer.

Ultimately, the redevelopment of the NSW Central Cancer Registry will result in the provision of up-to-date data on cancer incidence, mortality, prevalence and survival in flexible formats.

\section{REFERENCES}

1. NSW Department of Health. Central Cancer Registry business case for replacement Cancer Registry Systems. November 1997. Unpublished report.

2. Tracey E. Notification to the Central Cancer Registry-Outline of business requirements. Presented to the Patient Administration System (PAS) Consortium Meeting. December 7th 1999. Unpublished report.

3. CCR Specific Data items www.health.nsw.gov.au/iasd/dm/ isc/instructions/2000-2001/isc-instructions-2000-2001.html.

4. Health Information Exchange Cancer Feed Scope, version 1.11, October 2000.

5. Implementation of Health Level 7 (HL7) version 2.3.1 Part 4 Pathology results for registries. AS 4700.4 October 2000.

6. Tracey E. Resource requirements for processing the backlog of CCR notifications. May 2000. Unpublished report. W

\section{TOWARDS A CLINICAL CANCER INFORMATION SYSTEM}

Hanna Noworytko and Helen Moore

Epidemiology and Surveillance Branch

NSW Department of Health

\section{Bruce Armstrong}

Manager, Cancer Research and Registries

NSW Cancer Council
The efficient delivery of better health care depends on the availability of information on the outcomes of health care. The NSW Clinical Cancer Registries Project (ClinCRs) aims to improve — at a state level—-the collection, collation and analysis of data on patterns of cancer treatment and the health outcomes of cancer patients. 